\title{
Radiation dose of aircrews during a solar proton event without ground-level enhancement
}

\author{
R. Kataoka ${ }^{1,2}$, Y. Nakagawa ${ }^{3}$, and T. Sato ${ }^{4}$ \\ ${ }^{1}$ National Institute of Polar Research, 10-3 Midori-cho, Tachikawa, Tokyo 190-8518, Japan \\ ${ }^{2}$ Department of Polar Science, The Graduate University for Advanced Studies (SOKENDAI), 10-3 Midori-cho, Tachikawa, \\ Tokyo 190-8518, Japan \\ ${ }^{3}$ Ibaraki University, 2-1-1 Bunkyo, Mito, Ibaraki 310-8518, Japan \\ ${ }^{4}$ Japan Atomic Energy Agency, 2-4 Shirakata-Shirane, Tokai-mura, Ibaraki 319-1195, Japan \\ Correspondence to: R. Kataoka (kataoka.ryuho@nipr.ac.jp)
}

Received: 11 September 2014 - Revised: 6 December 2014 - Accepted: 8 December 2014 - Published: 15 January 2015

\begin{abstract}
A significant enhancement of radiation doses is expected for aircrews during ground-level enhancement (GLE) events, while the possible radiation hazard remains an open question during non-GLE solar energetic particle (SEP) events. Using a new air-shower simulation driven by the proton flux data obtained from GOES satellites, we show the possibility of significant enhancement of the effective dose rate of up to $4.5 \mu \mathrm{Sv} \mathrm{h}^{-1}$ at a conventional flight altitude of $12 \mathrm{~km}$ during the largest SEP event that did not cause a GLE. As a result, a new GOES-driven model is proposed to give an estimate of the contribution from the isotropic component of the radiation dose in the stratosphere during non-GLE SEP events.
\end{abstract}

Keywords. Interplanetary physics (cosmic rays; energetic particles)

\section{Introduction}

Solar energetic particles (SEPs) are sometimes energetic enough and the flux is high enough to cause air showers in the stratosphere and in the troposphere, which are an important ionization source in the atmosphere. The most energetic events can be observed even at ground-based neutron monitors, and are called ground-level enhancements (GLE) (Forbush, 1946). Recently, Matthia et al. (2009a) reported that the effective dose rate at a conventional flight altitude of $40 \mathrm{kft}$ $(\sim 12 \mathrm{~km})$ reached $1.0 \mathrm{mSv} \mathrm{h}^{-1}$ during the largest GLE-69 during solar cycle 23 . The total effective dose at flight altitude has been estimated for all of the GLE events during so- lar cycle 23 , and ranged from $2.0 \mu \mathrm{Sv}$ to $0.3 \mathrm{mSv}$ (Shea and Smart, 2012).

Kataoka et al. (2014) recently developed a forecast model called WASAVIES (Warning System of AVIation exposure to SEP) to give the time profile of effective dose rate at flight altitude during GLEs, using a new air-shower simulation developed by Sato et al. (2014). Based on the air-shower simulation, it was pointed out that SEP events may also contribute to aircrews' exposure; i.e., the flux of low-energy protons of an order of a few $100 \mathrm{MeV}$ can be large enough to cause a radiation dose at conventional flight altitude, even though the low-energy protons do not cause a GLE. The purpose of this study is to evaluate the effective dose rate at conventional flight altitude during such non-GLE SEP events, using the new air-shower simulation and proton flux data obtained from GOES satellites.

\section{Method of analysis}

According to the NOAA SEP list (http://umbra.nascom.nasa. gov/SEP/), there were only four non-GLE SEP events that exceeded $10^{4} \mathrm{pfu}$ (particles $\mathrm{cm}^{-2} \mathrm{~s}^{-1} \mathrm{sr}^{-1}$ ) at $\geq 10 \mathrm{MeV}$ protons, that did not cause GLEs. The SEP event on 9 November 2000, which was associated with an M7.4 flare, had the largest flux ( $\sim 70 \mathrm{pfu})$ at $80-165 \mathrm{MeV}$ protons among these top-four events (only 1.5 pfu on 24 March 1991, 0.3 pfu on 25 September 2001, and 0.06 pfu on 24 November 2001), and we focus on the 9 November 2000 non-GLE SEP event as the most plausible candidate for causing a significant radiation dose at conventional flight altitude. The well-known 

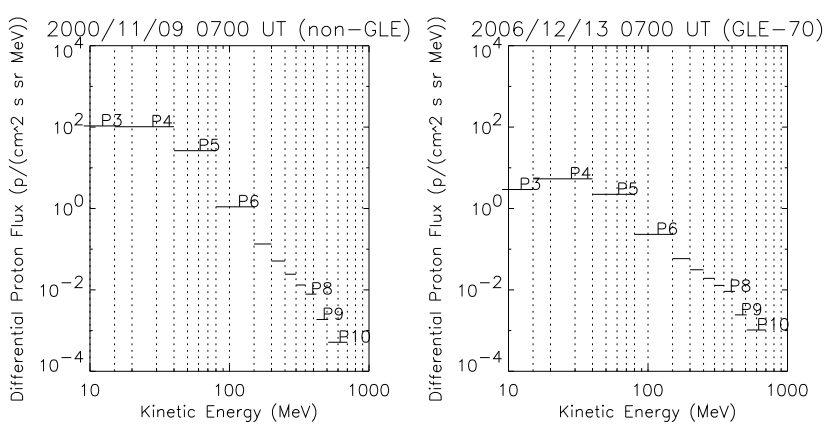

Figure 1. Examples of $5 \mathrm{~min}$ averaged proton spectra for the nonGLE SEP event (left) and the GLE-70 event (right). Dotted lines show the energy of the pre-calculated mono-energetic protons by Sato et al. (2014).
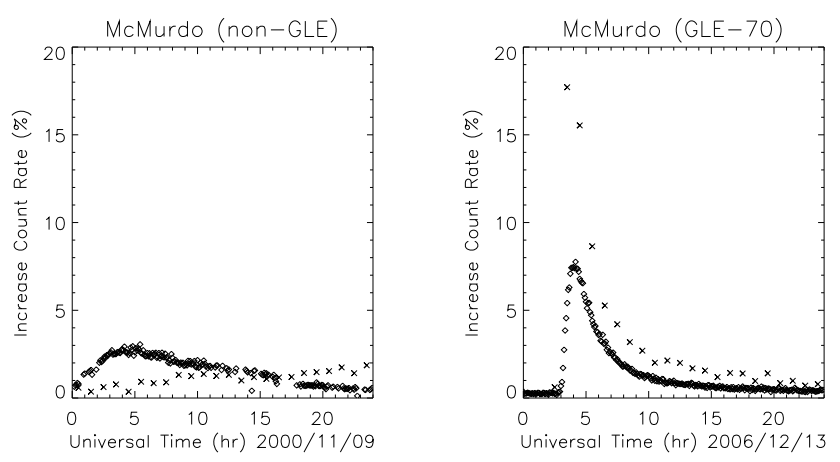

Figure 2. Simulated (diamonds) and observed (crosses) increase count rate at the McMurdo neutron monitor during the non-GLE SEP event (left) and during the GLE-70 event (right).

GLE-70 of 13 December 2006, which was the third-largest GLE event during solar cycle 23 (Shea and Smart, 2012), is also evaluated as a reference.

We use the simulation method developed by Sato et al. (2014), in which the radiation dose is calculated by using the response function generated for mono-energetic protons on the basis of an air-shower simulation performed with PHITS (Particle and Heavy Ion Transport code System) version 2.52 (Sato et al., 2013). INCL (The Liege Intranuclear Cascade model) version 4.6 (Boudard et al., 2013 ) is employed as the default nuclear reaction model for intermediate-energy neutrons in PHITS 2.52. The simulation method of Sato et al. (2014) is capable of quantitatively evaluating the altitude-dependent effective dose rate as well as the increase count rate at ground-based neutron monitors for both GLE events and for non-GLE events by preparing the input data as the proton spectra at the top of the atmosphere (TOA) of $86 \mathrm{~km}$ in altitude.

The proton spectra at TOA are calculated from the GOES11 proton flux data obtained at geosynchronous orbit (GEO) as follows. We assume that the ratio of proton fluxes at GEO and at TOA in the polar region as 0.4 as a common value for all of the energy ranges; i.e., we used the cor-
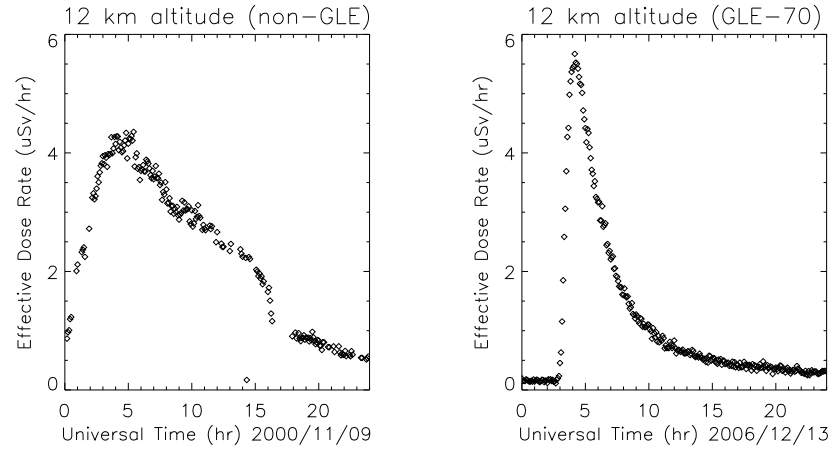

Figure 3. Simulated effective dose rate at $12 \mathrm{~km}$ altitude at the polar region during the non-GLE SEP event (left) and during the GLE-70 event (right).

rected proton flux of GOES-EPS P4 (15-44 MeV), P5 (40$80 \mathrm{MeV})$, and P6 (80-165 MeV) channels as the proton flux at TOA by simply multiplying the "penetration factor" of 0.4 . The GOES-EPS P7 $(165-500 \mathrm{MeV})$ channel is not employed because the assumption of constant flux for this relatively broad energy range may induce an inaccuracy in the calculation. Instead, we use the proton flux of the GOESHEPAD P8 (350-420 MeV), P9 (420-510 MeV), and P10 $(510-700 \mathrm{MeV})$ channels with the same penetration factor, and linearly interpolate the proton flux between the P6 and P8 channels on a log-log scale. The GOES-HEPAD P11 $(>700 \mathrm{MeV})$ channel is omitted because its upper limit energy is not specified. Examples of proton spectra at TOA for the non-GLE SEP event and the GLE-70 event are shown in Fig. 1. Note that the results shown in this paper can be used for a different penetration factor by linear scaling.

The assumed penetration factor of 0.4 is selected in this paper by finding the largest value not to cause a simulated GLE in the non-GLE SEP event on 9 November 2000, and to cause a simulated GLE in the GLE-70 event. In reality, however, the proton fluxes at GEO and at TOA do not have such a simple relationship (Bornebusch et al., 2010), and further careful investigations are needed to understand the actual balance between the mirroring and geometrical focusing of energetic protons in the complex geomagnetic field. Also, it has been shown that GLEs have an anisotropic component in their initial phase, and the dose rates at certain locations are expected to have been much higher than what is calculated in this work (see Matthia et al., 2009b; Meier and Matthia, 2014). It is therefore important to note that using the data from GOES alone cannot give a complete picture of the event and its impact over all longitudes and latitudes, and our method is limited to estimating the contribution from the isotropic component. 

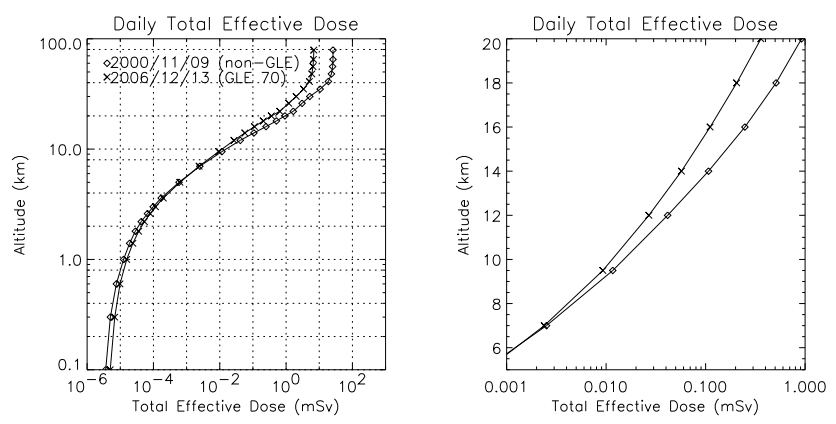

Figure 4. Simulated total effective dose at various altitudes during the non-GLE event (diamonds) and during the GLE-70 event (crosses) for $24 \mathrm{~h}$.

\section{Results}

Figure 2 shows the result of the comparison between simulated and observed increase count rates during the non-GLE SEP event on 9 November 2000 and the GLE-70 event on 13 December 2006 at McMurdo station. The increase count rate is defined in this paper as the neutron monitor count rate above the mean value of the previous day. The simulated increase count rate is an order of the approximate noise level of $100 \mathrm{cph}$ for the non-GLE SEP event. For the GLE-70 event, the increase count rate significantly exceeds the noise level, but it is lower than that of observed values, which is reasonable considering that we omitted the expected additional counts from very energetic particles of greater than $700 \mathrm{MeV}$ for this particular GLE-70 event.

Figure 3 shows the result of a simulated effective dose rate at $12 \mathrm{~km}$ altitude in the polar region during the non-GLE SEP and GLE-70 events. It is found that the effective dose rate during the non-GLE SEP event can be up to $4.5 \mu \mathrm{Sv} \mathrm{h}^{-1}$. This value is comparable to the maximum effective dose rate during the GLE-70 event, approximately $5.8 \mu \mathrm{Sv} \mathrm{h}^{-1}$, instead of the large difference in the increase count rates between the two events as shown in Fig. 2. As a reference value, it is also worthwhile to note that the ambient dose equivalent rate during solar minimum (2006-2008) was $\sim 9 \mathrm{uSv} \mathrm{h}^{-1}$ in the polar region at $41 \mathrm{~K}$ feet altitude (Meier et al., 2009).

Figure 4 shows $24 \mathrm{~h}$ integrated total effective doses during the non-GLE SEP and GLE-70 events. In order to consider the additional shielding due to airframe, we assume that the thickness of the airframe is $10 \mathrm{~g} \mathrm{~cm}^{-2}$, and we ignore the dose contribution from less than $100 \mathrm{MeV}$ protons. The total effective dose largely changes depending on the altitude, especially at around the conventional flight altitude; e.g., an increase in flight altitudes from 9.5 to $14 \mathrm{~km}$ results in an increase in the total effective dose by nearly 1 order of magnitude. The total effective dose of the non-GLE SEP event is found to be up to $0.03 \mathrm{mSv}$ at an altitude of $12 \mathrm{~km}$. The total effective dose for the non-GLE SEP event is more enhanced against a GLE event at higher altitude.
During one flight, the aircraft is at high latitudes and high altitudes only for a couple of hours. The calculated dose is only a theoretical value, and is not the actual radiation exposure of an aircrew during such an event. In order to estimate the total dose for a flight, one needs to take into account the flight durations and flight profiles.

\section{Summary and discussion}

We showed the possibility of significant enhancement of the radiation dose at conventional flight altitude during the largest SEP event that did not cause a GLE. It is important to note, however, that the SEP event on 9 November 2000 is the only such event we found so far using the GOES data after 1976. Such powerful non-GLE SEP events are therefore very rare, and rarer than GLEs.

As a next step, we extend this study to find similar but significantly weaker SEP events, and to evaluate the possible total impact on the atmosphere that may show dynamical change over solar cycles. This attempt gives a plausible altitude-dependent energy deposition rate by solar protons in the stratosphere, and the realistic limitation would contribute to understanding the fundamental mechanism for connecting the space environment and the planetary atmosphere.

Acknowledgements. We thank Terry Onsager of NOAA for his advice on analyzing the GOES data. GOES data are provided by NOAA/NGDC. Neutron monitors of the Bartol Research Institute are supported by NSF grant ATM-0000315. The production of this paper was supported by an NIPR publication subsidy. This work was supported by JSPS KAKENHI Grant Number 26106006.

Topical Editor K. Hosokawa thanks one anonymous referee for his/her help in evaluating this paper.

\section{References}

Bornebusch, J. P., Wissing, J. M., and Kallenrode, M.-B.: Solar particle precipitation into the polar atmosphere and their dependence on hemisphere and local time, Adv. Space Res., 45, 632-637, 2010.

Boudard, A., Cugnon, J., David, J. C., Leray, S., and Mancusi, D.: New potentialities of the Liege intranuclear cascade model for reactions induced by nucleons and light charged particles, Phys. Rev. C, 87, 014606, doi:10.1103/PhysRevC.87.014606, 2013.

Forbush, S. E.: Three unusual cosmic-ray increases possibly due to charged particles from the Sun, Phys. Rev., 70, 771-772, 1946.

Kataoka, R., Sato, T., Kubo, Y., Shiota, D., Kuwabara, T., Yashiro, S., and Yasuda, H.: Radiation dose forecast of WASAVIES during ground level enhancement, Space Weather, 12, 380-386, doi:10.1002/2014SW001053, 2014.

Matthia, D., Heber, B., Reitz, G., Meier, M., Sihver, L., Berger, T., and Herbst, K.: Temporal and spatial evolution of the solar energetic particle event on 20 January 2005 and resulting radiation doses in aviation, J. Geophys. Res., 114, A08104, doi:10.1029/2009JA014125, 2009a. 
Matthia, D., Heber, B., Reitz, G., Meier, M., Sihver, L., Berger, T., and Meier, M.: The ground level event 70 on December 13th 2006 and related effective doses at aviation altitudes, Radiat. Prot. Dosim., 136, 304-310, doi:10.1093/rpd/ncp141, $2009 \mathrm{~b}$.

Meier, M. M. and Matthia, D.: A space weather index for the radiation field at aviation altitudes, J. Space Weather Space Clim., 4, A13, doi:10.1051/swsc/2014010, 2014.

Meier, M. M., Hubiak, M., Matthia, D., Wirtz, M., and Reitz, G.: Dosimetry at aviation altitudes (2006-2008), Radiat. Prot. Dosim., 136, 251-255, doi:10.1093/rpd/ncp142, 2009.

Sato, T., Niita, K., Matsuda, N., Hashimoto, S., Iwamoto, Y., Noda, S., Ogawa, T., Iwase, H., Nakashima, H., Fukahori, T., Okumura, K., Kai, T., Chiba, S., Furuta, T., and Sihver, L.: Particle and Heavy Ion Transport Code System PHITS, Version 2.52, J. Nucl. Sci. Technol., 50, 913-923, 2013.
Sato, T., Kataoka, R., Yasuda, H., Seiji, Y., Kuwabara, T., Shiota, D., and Kubo, Y.: Air shower simulation for WASAVIES: Warning system for aviation exposure to solar energetic particles, Radiat. Prot. Dosim., 161, 274-278, doi:10.1093/rpd/nct332, 2014.

Shea, M. A. and Smart, D. F.: Space Weather and the Ground-Level Solar Proton Events of the 23rd Solar Cycle, Space Sci. Rev., 171, 161-188, 2012. 